\title{
Experimental observation of evanescent modes at the interface to slow-light photonic crystal waveguides
}

\author{
Marko Spasenović, ${ }^{1, *,+}$ Thomas P. White, ${ }^{2,+}$ Sangwoo Ha, ${ }^{2}$ Andrey A. Sukhorukov, ${ }^{2}$ Tobias Kampfrath, ${ }^{1}$ \\ Yuri S. Kivshar, ${ }^{2}$ C. Martijn de Sterke, ${ }^{3}$ Thomas F. Krauss, ${ }^{4}$ and L. (Kobus) Kuipers ${ }^{1}$ \\ ${ }^{1}$ Center for Nanophotonics, FOM Institute for Atomic and Molecular Physics (AMOLF), \\ Science Park 104, 1098 XG, Amsterdam, Netherlands \\ ${ }^{2}$ Nonlinear Physics Centre and Centre for Ultrahigh-Bandwidth Devices for Optical Systems (CUDOS), \\ Research School of Physics and Engineering, Australian National University, Canberra, ACT 0200, Australia \\ ${ }^{3}$ Centre for Ultrahigh-Bandwidth Devices for Optical Systems (CUDOS), Institute for Photonics \\ and Optical Sciences (IPOS) and School of Physics, University of Sydney, NSW 2006, Australia \\ ${ }^{4}$ School of Physics and Astronomy, University of St. Andrews, St. Andrews, Fife, KY169SS, UK \\ ${ }^{*}$ Corresponding author: marko@amolf.nl
}

Received February 3, 2011; accepted February 27, 2011; posted March 8, 2011 (Doc. ID 142044); published March 25, 2011

\begin{abstract}
We experimentally study the fields close to an interface between two photonic crystal waveguides that have different dispersion properties. After the transition from a waveguide in which the group velocity of light is $v_{g} \sim c / 10$ to a waveguide in which it is $v_{g} \sim c / 100$, we observe a gradual increase in the field intensity and the lateral spreading of the mode. We attribute this evolution to the existence of a weakly evanescent mode that exponentially decays away from the interface. We compare this to the situation where the transition between the waveguides only leads to a minor change in group velocity and show that, in that case, the evolution is absent. Furthermore, we apply novel numerical mode extraction techniques to confirm experimental results. (c) 2011 Optical Society of America OCIS codes: $130.5296,350.4238$.
\end{abstract}

Photonic crystal (PhC) waveguides that support slowlight modes present new opportunities for enhancing linear and nonlinear optical effects and realization of compact optical delay lines [1,2]. Waveguide dispersion and group velocity can be controlled precisely by modifying the $\mathrm{PhC}$ geometry to achieve broadband slow-light propagation [3] or other dispersive properties tailored for applications, such as tunable delays [4] or switching [5]. In order to utilize the benefits of slow light in $\mathrm{PhC}$ waveguides, light must be efficiently coupled to them.

Coupling to slow-light $\mathrm{PhC}$ modes was thought to be difficult [6], one reason being the mismatch in mode profiles between fast and slow light in PhCs. Theoretical work has demonstrated that evanescent modes can play a role in allowing efficient excitation of slow light [7]. Evanescent modes can be excited at interfaces with other waveguides, where they assist in matching the transverse mode profiles on either side of the interface [8]. It was predicted that the presence of a weakly evanescent mode near the inflection point of a $\mathrm{PhC}$ waveguide's dispersion curve allows better than $99 \%$ coupling efficiency from an incident mode with group velocity $v_{g} \sim c / 5$ to one with $v_{g} \sim c / 1000$ [7,, ] . More generally, evanescent modes play a crucial role in "frozen light" in which light can be perfectly coupled to zero group velocity modes [9]. A mathematical description of propagating and evanescent modes in $\mathrm{PhCs}$ is given in [10].

Here, we report experimental evidence for the existence of weakly evanescent modes at an interface to slow-light PhC waveguides. We show that, at low group velocities in these waveguides, the field intensity grows and the lateral extent of the electric field increases gradually into the slow-light waveguide, while this behavior is absent at higher group velocities. Both the gradual rise in intensity and the evolution of the field profile are signatures of a weakly evanescent mode. A novel numerical mode extraction technique [11] confirms these conclusions and reveals an evanescent mode that decays over a length of $\sim 10 \mathrm{PhC}$ periods.

The PhC consisted of three waveguide sections: an $80 \mu \mathrm{m}$ long central waveguide supporting a mode with a dispersion inflection point, at which $v_{g}<c / 100$, connected at both ends to 10 periods of modified waveguide. For the frequencies used in this investigation, the end waveguides supported a relatively fast mode, with group velocity $v_{g} \approx c / 5$. The outer PhC sections were connected to tapered ridge access waveguides. The samples were fabricated on a silicon-on-insulator wafer consisting of a $220 \mathrm{~nm}$ Si layer on a $2 \mu \mathrm{m}$ layer of silica using electron beam lithography and reactive ion etching. The PhCs were underetched using hydrofluoric acid to form symmetric Si membranes in air. The geometry is illustrated in Fig. 1(a). Only one end of the structure is shown, the other being the mirror image. The $\mathrm{PhC}$ waveguides were formed in a triangular lattice of holes with period $d=$ $420 \mathrm{~nm}$ and hole radius $r=128 \mathrm{~nm}=0.305 d$. To create an inflection point in the dispersion curve, we locally modified the geometry by shifting the first two rows of holes on either side of the waveguide in a direction parallel to the waveguide. The first row of holes was displaced by $p_{1}=0.3 d$, and the second row by $p_{2}=0.4 d$ in the same direction, as illustrated in Fig. 1(a). The $\mathrm{PhC}$ sections at either end were similar to the central section, but the lattice was "stretched" by $30 \mathrm{~nm}$ per unit cell along the waveguide so that the wavelength of the inflection point in the central section coincided with a fast mode in the end sections. A similar approach was used in [7] to demonstrate efficient coupling between fast and slow PhC modes.

The waveguide was characterized by obtaining transmission and group velocity spectra. The group velocity 

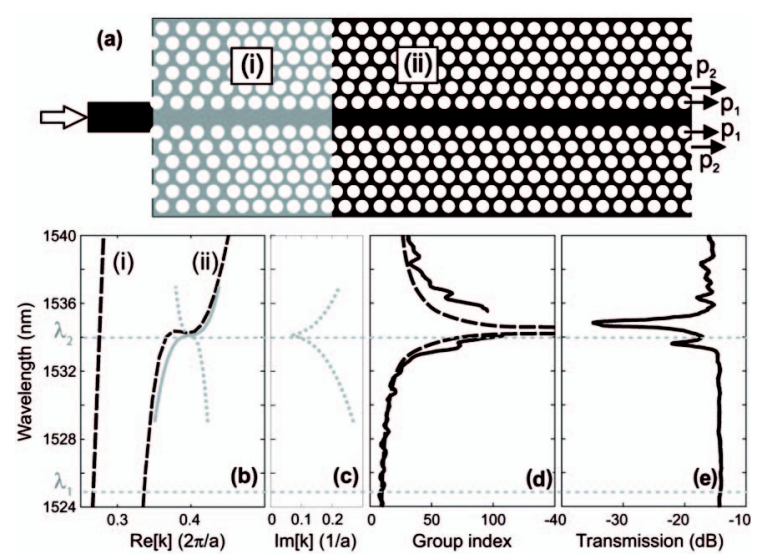

Fig. 1. (a) Geometry of the waveguide. In section (i), the lattice was stretched by $30 \mathrm{~nm}$. (b) Calculated dispersion for the two waveguide sections (dashed black curves) and dispersion retrieved from the numerical fit for propagating and evanescent modes (solid and dashed gray curves, respectively). The black curve was calculated for an infinitely long waveguide. (c) Imaginary component of the wave vector of the evanescent mode. (d) Calculated (dashed curve) and measured (solid curve) group index spectrum. (e) Measured transmission spectrum.

was measured using Fourier transform interferometry [12]. The measured transmission and group index are shown in Figs. 1(d) and 1(e), respectively. We attribute the sharp drop in transmission in the slow-light region to increased propagation loss rather than inefficient coupling to the slow-light mode. The dispersion curves for the two waveguide sections, obtained by fitting threedimensional band structure calculations to the measurements, are shown in Fig. 1(b). The group index obtained by the same calculation is shown in Fig. 1(d). At a freespace wavelength of $\lambda=1533.95 \mathrm{~nm}$, indicated by a horizontal dashed gray line, the dispersion of section (ii) of the waveguide displays an inflection point, with a correspondingly high group index $n_{g} \sim 100$. At the same wavelength, the dispersion curve in section (i) is steep, resulting in a relatively low group index $n_{g} \sim 5$. On the other hand, at $\lambda=1525 \mathrm{~nm}$, indicated by the other dashed gray line in Fig. $1, n_{g} \sim 5$ in the outer sections and $n_{g} \sim 10$ in the central section. We study the interface between sections (i) and (ii) at these two wavelengths.

We perform phase- and polarization-sensitive nearfield optical measurements [13] on the waveguide, which can separate forward and backward-propagating Bloch modes [14]. Figure 2 depicts $\left|E_{x}\right|$, the modulus of the transverse component of the electric field, i.e., the component that oscillates perpendicular to the waveguide, as a function of position (in units of $d$ ). Light propagates from left to right. The measurement of Fig. 2(a) was taken at $\lambda_{1}=1525 \mathrm{~nm}$. At this wavelength, the group velocity in the central waveguide is $v_{g} \sim c / 10$. The measurement of Fig. 2(b) was taken at $\lambda_{2}=1533.95 \mathrm{~nm}$. At this wavelength, the group velocity in the central waveguide is $v_{g} \sim c / 100$. The amplitude was normalized in both images such that the maximum amplitude in the first waveguide is equal to 1 .

We clearly observe different behavior of the electric field in the two cases. Close to the inflection point, the electric field amplitude grows into the slow-light section by a factor of $\sim 5$, as expected [8]. Far from the inflection

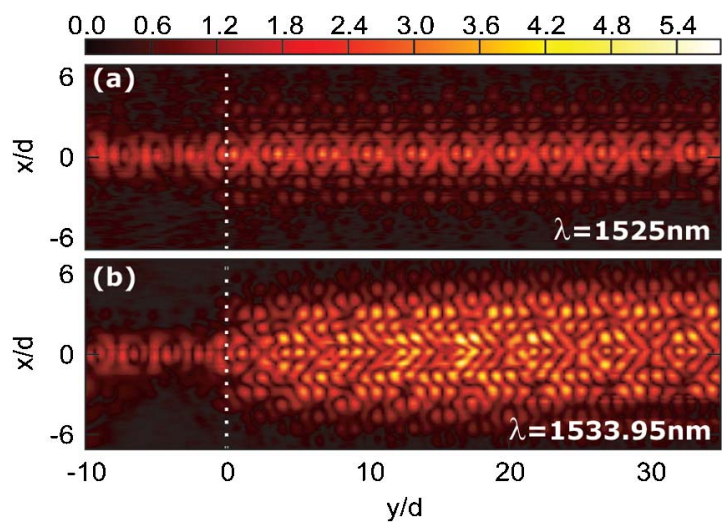

Fig. 2. (Color online) Near-field measurements of the electric field above the waveguide. (a) Light is "fast" in both waveguide sections, at a wavelength of $\lambda_{1}=1525 \mathrm{~nm}$. (b) Light is slow in the second section, at $\lambda_{2}=1533.95 \mathrm{~nm}$. The dashed white line indicates the interface between the two waveguide sections.

point, it grows by less than $10 \%$. Another difference is that, far from the inflection point, the electric field does not expand laterally after entering the central waveguide. To the contrary, close to the dispersion inflection point, the field laterally expands further into the $\mathrm{PhC}$ lattice. A deeper penetration of the field into the surrounding crystal is indeed expected for slow light; what is striking here is that the expansion grows over several tens of periods, which indicates an evanescently decaying mode. A beating with a period of five lattice constants is evident in both images due to the interference between forwardand backward-propagating Bloch modes.

The integrated intensity $\int\left|E_{x}^{2}\right| \mathrm{d} x$ is plotted in Fig. 3(a) as a function of position along the waveguide, $y$. The solid vertical line indicates the interface between the two $\mathrm{PhC}$ sections. In the first 15 periods beyond the interface, intensity at $\lambda_{2}$ grows to a value about ten times higher than the intensity at $\lambda_{1}$, consistent with the measured slowdown factor of 10 . The rise in intensity was pointed out in [8], based on numerics. The modes interfere destructively at the interface, and, since one is evanescent, further into the waveguide only the propagating mode remains, resulting in a field strength that is higher further down the waveguide than at the interface. As the light
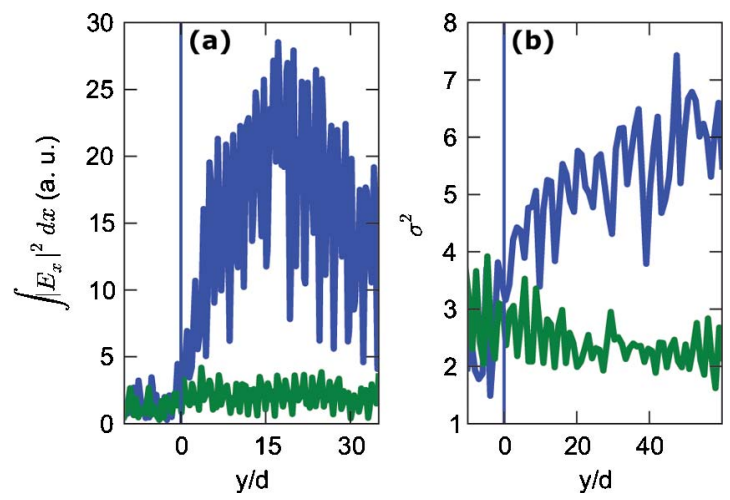

Fig. 3. (Color online) Intensity and field spread. (a) $\int\left|E_{x}\right|^{2} \mathrm{~d} x$ along the length of the waveguide. The green (bottom) curve is measured at $\lambda_{1}=1525 \mathrm{~nm}$; the blue (top) curve is measured at $\lambda_{2}=1533.95 \mathrm{~nm}$. (b) Variance of the intensity along the waveguide. 
propagates even further, the measured intensity starts to decay, due to propagation losses $[15,16]$.

To quantitatively study the broadening of the mode profile in the case of coupling to slow light, we determine the variance of the intensity along the length of our structure. The variance is defined as

$$
\sigma^{2}=\frac{\int(x-\mu)^{2} I(x) \mathrm{d} x}{\int I(x) \mathrm{d} x} .
$$

Here, $I(x)$ is the measured intensity and $\mu$ is the center of mass on the $x$ axis. The variance shows directly how much the intensity spreads into the surrounding $\mathrm{PhC}$ lattice. The variance is depicted in Fig. 3(b). To avoid effects due to the interference of different Bloch harmonics, the variance is averaged over each unit cell.

At $\lambda_{1}=1525 \mathrm{~nm}$, the variance does not change noticeably over the length of the waveguide. At $\lambda_{2}=$ $1533.95 \mathrm{~nm}$, however, the mode expands progressively as it propagates further into the second waveguide, with the variance growing by a factor of $\sim 2$. Both the gradual expansion of the mode and the rise in intensity are signatures of a weakly evanescent mode excited at the interface between the fast and slow sections of the waveguide.

To retrieve the dispersion of the evanescent mode, we perform a series of near-field measurements at different wavelengths. We apply a numerical dispersion extraction technique [11] to find the optimal combination of propagating and evanescent Bloch modes (solid and dashed gray lines in Fig. 1(b)]. As pointed out in [8], at the inflection point, the evanescent and propagating modes are phase matched. The imaginary component of the wave vector [Fig. 1(c)] contains a dip centered at the inflection point. There, the evanescent mode decays only weakly, with a decay length of $\sim 10$ periods, and assists in the coupling to slow light [8], consistent with the measured gradual spread of the mode and rise in intensity.

In conclusion, we have used a combination of direct near-field measurements and dispersion extraction techniques to observe experimentally the excitation of a weakly evanescent mode at the interface between two $\mathrm{PhC}$ waveguides supporting propagating modes with very different group velocities. Such evanescent modes have been shown theoretically to enable very efficient coupling between fast and slow light, and also play a crucial role in the phenomenon of frozen light. This work provides a framework for further experimental studies of frozen light behavior in PhCs.

This work is part of the research program of the Stichting voor Fundamenteel Onderzoek der Materie (FOM), which is financially supported by the Nederlandse Organisatie voor Wetenschappelijk Onderzoek (NWO). We thank the EC Marie Curie Scheme (contract MEST-CT2005-021000). This work was produced with the assistance of the Australian Research Council under its ARC Centres of Excellence Program.

'These authors contributed equally to this work.

\section{References}

1. T. F. Krauss, Nat. Photon. 2, 448 (2008).

2. T. Baba, Nat. Photon. 2, 465 (2008).

3. J. Li, T. P. White, L. O. Faolain, A. Gomez-Iglesias, and T. F. Krauss, Opt. Express 16, 6227 (2008).

4. T. Baba, T. Kawasaki, H. Sasaki, J. Adachi, and D. Mori, Opt. Express 16, 9245 (2008).

5. T. Kampfrath, D. M. Beggs, T. P. White, M. Burresi, D. van Oosten, T. F. Krauss, and L. Kuipers, Appl. Phys. Lett. 94, 241119 (2009).

6. Y. A. Vlasov and S. J. McNab, Opt. Lett. 31, 50 (2006).

7. J. P. Hugonin, P. Lalanne, T. P. White, and T. F. Krauss, Opt. Lett. 32, 2638 (2007).

8. T. P. White, L. C. Botten, C. M. de Sterke, K. B. Dossou, and R. C. McPhedran, Opt. Lett. 33, 2644 (2008).

9. J. Ballato, A. Ballato, A. Figotin, and I. Vitebskiy, Phys. Rev. E 71, 036612 (2005).

10. L. C. Botten, T. P. White, A. A. Asatryan, T. N. Langtry, C. M. de Sterke, and R. C. McPhedran, Phys. Rev. E 70, 056606 (2004).

11. S. Ha, A. A. Sukhorukov, K. B. Dossou, L. C. Botten, C. M. de Sterke, and Y. S. Kivshar, Opt. Lett. 34, 3776 (2009).

12. A. Gomez-lglesias, D. O'Brien, L. O'Faolain, A. Miller, and T. F. Krauss, Appl. Phys. Lett. 90, 261107 (2007).

13. M. Burresi, R. J. P. Engelen, A. Opheij, D. van Oosten, D. Mori, T. Baba, and L. Kuipers, Phys. Rev. Lett. 102, 033902 (2009).

14. H. Gersen, T. J. Karle, R. J. P. Engelen, W. Bogaerts, J. P. Korterik, N. F. van Hulst, T. F. Krauss, and L. Kuipers, Phys. Rev. Lett. 94, 123901 (2005).

15. S. Mazoyer, P. Lalanne, J. C. Rodier, J. P. Hugonin, M. Spasenović, L. Kuipers, D. M. Beggs, and T. F. Krauss, Opt. Express 18, 14654 (2010).

16. S. Hughes, L. Ramunno, J. F. Young, and J. E. Sipe, Phys. Rev. Lett. 94, 033903 (2005). 